East African Medical Journal Vol. 87 No. 4 April 2010

SPECIES DISTRIBUTION AND ANTIFUNGAL SENSITIVITY PATTERNS OF VAGINAL YEASTS

F. Mutua, MBChB, MSc, Lecturer, Department of Medical Microbiology, College of Health Sciences, University of Nairobi, P.O. Box 19676 - 00202, Nairobi, Kenya, G. Revathi, MBBS. MD (Clin. Micro.) Professor, Department of Microbiology, Aga Khan University Hospital, Nairobi, P.O. Box 30270 - 00100, Nairobi, J. M. Machoki, MBChB, MMed, Fellowship in Anthropob, Senior Lecturer, Department of Obstetrics and Gynaecology, College of Health Sciences, University of Nairobi, P.O. Box 19676 -00202, Nairobi, Kenya

Request for reprints to: Dr. F. Mutua, Department of Medical Microbiology, College of Health Sciences, University of Nairobi, P.O. Box 19676-00202, Nairobi, Kenya

\title{
SPECIES DISTRIBUTION AND ANTIFUNGAL SENSITIVITY PATTERNS OF VAGINAL YEASTS
}

\author{
F. MUTUA, G. REVATHI and J.M. MACHOKI
}

\begin{abstract}
Objectives: To identify yeast isolates in vaginal specimens to species level and determine their antifungal susceptibility patterns.

Design: Cross-sectional laboratory-based study.

Setting: The Aga Khan University Hospital (AKUH), Nairobi.

Subjects: Yeast isolates from high vaginal swabs presented to the laboratory for culture and sensitivity were identified to species level using the API Candida system and subjected to broth microdilution susceptibility testing.

Main outcome measures: Frequency tables and graphs were used to summarise the data. Susceptibility data was analysed by the non-parametric Fisher's exact test. $P<0.05$ was considered statistically significant.

Results: A total of 101 yeasts were studied. C.albicans was the prominent species $(69.3 \%)$ followed by C. glabrata (12.9\%), C. famata (5.0\%), C. krusei (3.0\%), Trichosporon species $(3.0 \%)$ and S.cerevisiae $(3.0 \%)$, C.parapsilosis $(1.0 \%)$. Three $(3.0 \%)$ of the isolates had profiles that fell between $C$. glabrata and $C$. famata. The percentages of $C$. albicans susceptible to flucytosine, amphotericin B, fluconazole and itraconazole were 94.3, 92.9, 100 and 90 respectively; that of non-albicans isolates were 93.5, 80.6, 77.4 and 29 respectively. There was no significant difference $(p>0.05)$ between the susceptibility of C.albicans and non-albicans isolates to flucytosine and amphotericin $B$, however there was a significant difference $(p<0.05)$ to fluconazole and itraconazole.

Conclusions: $C$. albicans was the predominant cause of vulvovaginal candidiasis in this study, and demonstrated good susceptibility to antifungal agents tested. A significant number of non-albicans yeasts were identified; these demonstrated reduced susceptibility to all drugs, in particular to the azoles which are commonly used for treatment of vaginal candidiasis. The isolation of non-albicans yeasts may have clinical implication given their reduced susceptibility to antifungals.
\end{abstract}

\section{INTRODUCTION}

Vulvovaginitis is the one of the most common gynaecological condition seen in women worldwide. The three common vaginal infections include vulvovaginal candidiasis (VVC), bacterial vaginosis, and Trichomonas vaginalis infection; there is a paucity of data on the global and national burden of these infections.

Vulvovaginal candidiasis frequently results from an overgrowth of the normal flora Candida species in the vagina. Candida is usually present in the lower female genital tract in small amounts, being part of the normal flora in $10-20 \%$ of healthy asymptomatic women (1). The over growth of the yeast and development of vulvovaginitis is usually prevented by the balance created by bacteria such as Lactobacillus acidophilus. Any condition that disrupts this balance of the normal flora predisposes to VVC. These include antibiotic use, oral contraceptives and contraceptive devices, high oestrogen levels and immunocompromised states such as diabetes mellitus and HIV.

C. albicans is responsible for most cases of VVC $(2,3)$; the rest are caused by non-albican species of Candida which include C. glabrata, C. krusei, and C. tropicalis. Non-albican species of Candida have demonstrated decreased susceptibility to 
commonly used antifungal agents (5). C.krusei has intrinsic resistance while C. glabrata has decreased susceptibility to fluconazole. C. albicans has also been shown to be developing resistance to the azole group of antifungal agents. This makes identification of yeasts to species level and antifungal susceptibility testing necessary in order to appropriately select the agent to use. Antifungal susceptibility testing may be used to predict clinical response, or at least to forecast treatment failure, and consequently can be used to develop local antibiograms to aid in empirical selection of antifungals; in testing of isolates to aid in selection of long-term therapy, and in testing of isolates from recurrent mucosal disease to aid in selection of alternative regimens.

Species identification and antifungal drug sensitivity profiles are not routinely carried out in the management of vaginal discharge among women seeking medical care in health facilities in Kenya. Therefore the present species distribution patterns and status of fungal resistance to conventional antifungal drugs among women in Kenya is largely unknown. This cross-sectional laboratory-based study was therefore designed to determine the species of yeasts causing vaginal infection and the current antifungal susceptibility patterns in the study set up. Documentation of a significant level of non-albicans species of Candida and their susceptibility patterns would pave the way for larger studies that would inform on any policy changes required in the of vaginal candidiasis.

\section{MATERIALS AND METHODS}

Study area: The study was carried out at the Microbiology laboratory of the Aga Khan University Hospital, a private university-affiliated hospital in Nairobi. The laboratory is well equipped and receives laboratory specimens from inpatients, outpatients from the casualty and outpatient clinics, as well as patients seeking medical care from the private doctors' clinics in the private wing.

Study population: All vaginal yeasts isolated at the Microbiology laboratory during the study period.

Samplesize: The minimum desired number of samples to be analysed was 100 .

Sampling: Consecutive sampling was used to select the vaginal yeasts for processing.

Inclusion criteria: Yeasts that demonstrated moderate or heavy growth on Sabouraud dextrose agar (SDA) were included in the study. In those with light growth, the gram stain from the original specimen was examined and only those with pus cells $\geq 2+$ were included in the study.
Laboratory procedures: Suspected yeast colonies were examined in a drop of normal saline for budding cells, and confirmed by the Gram stain. The yeast isolates were identified to species level using $\mathrm{API}^{\circledR}$ Candida system (BioMerieux, Marcy-l 'Etoile, France). The API Candida strip consists of ten tubes containing dehydrated substrates, which enabled the performance of sugar acidification tests and enzymatic reactions. The reactions produced during incubation were revealed by spontaneous color changes which were transformed into a numerical profile using the interpretation table provided. The numerical profile obtained was compared to those given in the profile list in the package insert to get the yeast species.

The susceptibility of the 101 yeast isolates to four antimycotics was tested using the broth microdilution method usingATB®Fungus2INT (BioMerieux, MarcyI'Etoile, France). The kit contains the following drug dilution ranges: amphotericin $\mathrm{B}$ (AMB) $0.5-16 \mathrm{mgl}^{-1}$, 5-flucytosine (5FC) $0.5-64 \mathrm{mgl}^{-1}$, fluconazole (FCA) $0.25-128 \mathrm{mgl}^{-1}$ and itraconazole (ITR) $0.125-4 \mathrm{mgl}^{-1}$. The strip consists of 16 pairs of cupules. The first pair does not contain any antifungal agent and is used as a positive growth control. The next 15 pairs contain the four antifungal agents at several concentrations, enabling minimum inhibitory concentration (MIC) determination. The preparation of the inoculums, strip inoculation and incubation, and reading of the results were carried out according to the manufacturer's instructions. The growth score was read for each cupule and the MIC determined for each drug as per instructions. The MICs were interpreted into clinical guidelines, sensitive, intermediate and resistant, by using Clinical and Laboratory Standards Institute(CLSI) recommended breakpoints (in $\mu \mathrm{gml}^{-1}$ ) for Candida species, provided in the manufacturer's instructions.

Data management: All data collected from the laboratory procedures were entered into MS Excel, verified and cleaned. Statistical analysis was performed using Statistical Package forSocial Sciences (SPSS) for Windows, version 12.0 (SPSS Inc., Chicago, IL, USA). Frequency tables and graphs were used to summarize the data. The susceptibility data was analyzed by the non-parametric Fisher's exact test. $\mathrm{P}<0.05$ was considered statistically significant.

\section{RESULTS}

Species identification and distribution: A total of 101 yeast isolates were collected and tested between November 2006 and January 2007 (Table 1). Candida species accounted for $94.1 \%$ of the isolates with C.albicans being the most common (73.7\% of all Candida species) (Figure 1).Among thenon-albicans species, C.glabrata (13.7\% of all Candida species) and C.famata (5.3\%) were the most commonly identified species. Three of 
the non-albicans isolates had profiles that fell between C. glabrata and C. famata. Six isolates were identified as non-Candida yeasts (Figure 2).

Table 1

Species distribution of vaginal yeasts isolated from women with vaginal discharge at the AKUH

\begin{tabular}{llc}
\multirow{2}{*}{ Specie } & \multicolumn{2}{l}{ Isolates } \\
& No. $(\%)$ \\
\hline C.albicans & 70 & 69.3 \\
C.glabrata & 13 & 12.9 \\
C.famata & 5 & 5.0 \\
C.krusei & 3 & 3.0 \\
C.parapsilosis & 1 & 1.0 \\
Unidentified Candida species* & 3 & 3.0 \\
Trichosporon species & 3 & 3.0 \\
Saccharomyces cerevisia & 3 & 3.0 \\
\hline
\end{tabular}

${ }^{*}$ Numerical profile could not differentiate between C.glabrata and C.famata

Figure 1

Percentage distribution of Candida species isolated from women with vaginal discharge at the AKUH

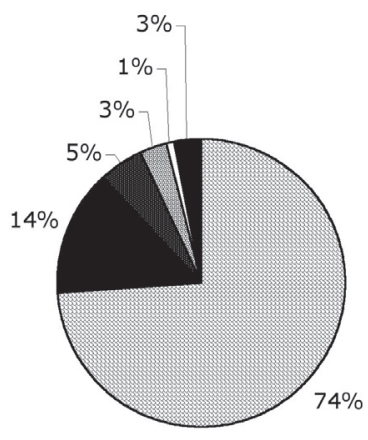

$$
\begin{aligned}
& \text { C. Albicans } \\
& \text { C. glabrata } \\
& \text { C. famata } \\
& \text { C. krusei } \\
& \square \text { C. parapsilosis } \\
& \text { Unidentified Candida } \\
& \text { Spp** }
\end{aligned}
$$

** Numerical profiles could not differentiate between C. glabrata and C. famata

Figure 2

Percentage distribution of yeast species isolated from women with vaginal discharge at the AKUH
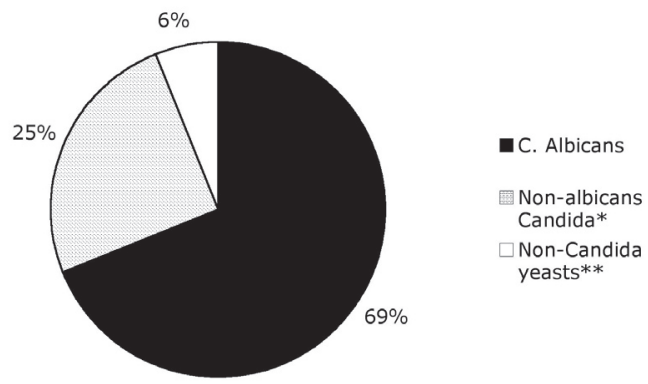

* C. glabrata, C. Famata, C. krusei, C. Parapsilosis, unidentified Candida species (C.glabrata/ C.famata)

** Trichosporon species, S. cerevisiae
Antifungal susceptibility testing: The 101 isolates were tested for susceptibility to flucytosine, amphotericin $\mathrm{B}$, fluconazole and itraconazole. Overall, 55 of the 70 C. albicans (78.6\%), six of the 25 non-albicans Candida strains (24\%) and one of the six strains of non-candidal yeasts $(16.7 \%)$ were susceptible to all tested antifungal agents. Resistance to one drug was demonstrated by $20 \%$ of the C. albicans strains (amphotericin B or fluconazole or itraconazole), $52 \%$ of the non-albicans Candida (flucytosine or itraconazole) and $66.7 \%$ of the non-candidal yeasts (amphotericin B or itraconazole). Of the $C$. albicans strains, $1.4 \%$ showed resistance to two of the tested agents (amphotericin Band itraconazole) while $8 \%$ of the non-albicans Candida and $16.7 \%$ of the non-candidal yeasts showed resistance to three of the antifungal agents (amphotericin B, fluconazole and itraconazole). No isolate tested was resistant to all tested antifungal agents. The percentage of resistant strains to each of the antifungal agents was as shown in Table 2.

Ninety five of the 101 yeast isolates $(94.1 \%)$ were susceptible to flucy tosine. One isolate $(1.0 \%)$ showed intermediate susceptibility and five isolates $(5.0 \%)$ were resistant. Sixty six of the 70 C.albicans strains (94.3\%), 23 of the 25 non-albicans Candida (92\%) and all the non-candidal yeasts were susceptible. The susceptibility patterns to flucytosine did not differ significantly between the $C$. albicans and the other yeast isolates ( $p=0.09)$ (Table 3). Of the 101 isolates, $90(89.1 \%)$ were susceptible to amphotericin B and $11(10.9 \%)$ resistant. Sixty five $(92.9 \%)$ of C. albicans strains, $23(92 \%)$ of the non-albicans Candida and two $(33.3 \%)$ of the non-candidal yeasts were susceptible. There was no significant difference between the susceptibility patterns of C. albicans and the nonalbican isolates $(\mathrm{p}=1.00)$ (Table 3).

Ninety four of the isolates $(93.1 \%)$ were susceptible to fluconazole. Four isolates $(4.0 \%)$ were susceptible-dose dependent while three (3.0\%) were resistant. All C. albicans strains were susceptible; 19 $(76 \%)$ of the non-albicans Candida and five $(83.3 \%)$ of the non-Candida yeasts were also susceptible. One of the three C. krusei strains was susceptible; the other two were susceptible-dose dependent. This is however not an important finding clinically since the species must be considered clinically resistant to fluconazole as per CLSI recommendations (6). There was significant difference between the susceptibility patterns of $C$. albicans and the other yeast isolates to fluconazole $(\mathrm{p}=0.00)$ (Table 3).

Itraconazole demonstrated the lowest susceptibility overall with only 72 of the 101 isolates $(71.3 \%)$ being susceptible, 23 isolates $(22.8 \%)$ were resistant. C.albicans was the species with the highest susceptibility at $90 \%$; the lowest was demonstrated by C. parapsilosis $(0 \%)$, C. glabrata $(15.4 \%)$ and C. famata $(20 \%)$. There was significant difference between the susceptibility patterns of $C$. albicans and the other yeast isolates to itraconazole $(\mathrm{p}=0.00)$ (Table 3$)$. 
Table 2

In vitro susceptibilities of 101 vaginal yeasts to four antifungal agents as determined by $M_{1} C_{90}$

\begin{tabular}{|c|c|c|c|c|}
\hline Drug and breakpoint & & C. albicans & $\begin{array}{l}\text { Species } \\
\begin{array}{l}(0=70) \quad \text { Non-albicans } \\
\text { Candida }(\mathrm{n}=25)\end{array}\end{array}$ & $\begin{array}{l}\text { Non- candidal } \\
\text { yeasts }(n=6)\end{array}$ \\
\hline \multirow{2}{*}{$\begin{array}{l}\text { Flucytosine } \\
(\mathrm{MIC}>16)\end{array}$} & $\mathrm{MIC}_{90}$ & 2 & 4 & 4 \\
\hline & $\%$ resistant & 5.7 & 4 & 0 \\
\hline Amphotericin B & $\mathrm{MIC}_{90}$ & 0.5 & 1 & 16 \\
\hline$(\mathrm{MIC} \geq 2)$ & $\%$ resistant & 7.1 & 8 & 66.7 \\
\hline Fluconazole & $\mathrm{MIC}_{90}$ & 1 & 32 & 128 \\
\hline$(\mathrm{MIC}>32)$ & $\%$ resistant & 0 & 8 & 16.7 \\
\hline Itraconazole & $\mathrm{MIC}_{90}$ & 0.125 & 4 & 4 \\
\hline$(\mathrm{MIC}>0.5)$ & $\%$ resistant & 10 & 56 & 33.3 \\
\hline
\end{tabular}

$\mathrm{MIC}_{90}$ and MIC in $\mu \mathrm{gm}^{-1}$

Table 3

Antifungal sensitivity patterns of vaginal yeasts isolated from women with vaginal discharge at the AKUH to amphotericin $B$, flucytosine, fluconazole and itraconazole

\begin{tabular}{|c|c|c|c|}
\hline \multirow{2}{*}{$\begin{array}{l}\text { Variable } \\
\text { (Drug) }\end{array}$} & \multicolumn{2}{|c|}{ Species } & \multirow{2}{*}{$\begin{array}{l}\text { Statistical tests } \\
\text { Fisher Exact test }\end{array}$} \\
\hline & C. albicans & Non albicans & \\
\hline \multicolumn{4}{|l|}{ Amphotericin $^{\mathrm{B}}$} \\
\hline Sensitivea & $92.9 \%$ & $80.6 \%$ & $X^{2}=3.30: 1 \mathrm{df}$ \\
\hline Resistant $^{\mathrm{B}}$ & $7.1 \%$ & $19.4 \%$ & $P>0.05(0.09)$ \\
\hline \multicolumn{4}{|l|}{ Flucytosine } \\
\hline Sensitivec & $94.3 \%$ & $93.5 \%$ & $X^{2}=0.02: 1 \mathrm{df}$ \\
\hline Intermediated \& re & $5.7 \%$ & $6.5 \%$ & $\mathrm{P}>0.05(1.00)$ \\
\hline \multicolumn{4}{|c|}{ Fluconazole } \\
\hline Sensitive ${ }^{f}$ & $100.0 \%$ & $77.4 \%$ & $X^{2}=16.98: 1 \mathrm{df}$ \\
\hline S-DDg \& resistant ${ }^{\mathrm{h}}$ & $0.0 \%$ & $22.6 \%$ & $\begin{array}{l}\mathrm{P}<0.05 \text { (0.00) } \\
\text { significant }\end{array}$ \\
\hline \multicolumn{4}{|l|}{ Itraconazole } \\
\hline Sensitive $^{\mathrm{i}}$ & $90.0 \%$ & $29.0 \%$ & $X^{2}=39.02: 1 \mathrm{df}$ \\
\hline S-DD \& Resistant ${ }^{\mathrm{K}}$ & $10.0 \%$ & $71.0 \%$ & $\begin{array}{l}\mathrm{P}<0.05 \text { (0.00) } \\
\text { significant }\end{array}$ \\
\hline
\end{tabular}

$\mathrm{S}-\mathrm{DD}=$ Susceptible-dose dependent

aMIC $<2 \mu \mathrm{gml}^{-1},{ }^{\mathrm{b}} \mathrm{MIC} \geq 2 \mu \mathrm{gml}^{-1},{ }^{\mathrm{c}} \mathrm{MIC} \leq 4 \mu \mathrm{gml}^{-1},{ }^{\mathrm{d}} \mathrm{MIC}=8-16 \mu \mathrm{gml}^{-1},{ }^{\mathrm{e}} \mathrm{MIC} \geq 32 \mu \mathrm{gml}^{-1},{ }^{\mathrm{f}} \mathrm{MIC}^{2} \leq 8 \mu \mathrm{gml}^{-1}$, ${ }^{\mathrm{g}} \mathrm{MIC}=16-32 \mu \mathrm{gmr}^{-1},{ }^{\mathrm{h}} \mathrm{MIC}^{2} \geq 64 \mu \mathrm{gmr}^{-1},{ }^{\mathrm{i}} \mathrm{MIC} \leq 0.125 \mathrm{MMI}^{-1}{ }^{\mathrm{MIC}}=0.25-0.5 \mu \mathrm{gml}^{-1},{ }^{\mathrm{k}} \mathrm{MIC}^{-1} 1 \mu \mathrm{gml}^{1}$

\section{DISCUSSION}

Most $(69.3 \%)$ of the species isolated in this study were C.albicans and a significant percentage (30.7\%) were non-albicans yeasts (Table 1 ). The non-albicans species isolated were mainly C.glabrata $(12.9 \%)$ and C. famata (5.0\%). Only two species of non-candida. yeasts were isolated: Trichosporon species and S.cerevisiae. C.albicans was the predominant species isolated in other studies (7-14) which concurs with the findings in this study. The distribution of the non-albicans species, however, differed in different studies. The most commonly isolated non-albicans species differed as C.glabrata (7-13) and C. parapsilosis (14). The second most common non-albicans species differed in the different studies as C.glabrata (14), C. tropicalis $(8,10,11)$ C. parapsilosis (12), C. famata (9) and C.krusei (7). 
Comparing the findings in the current study with those of some of the studies which demonstrated C.albicans in $40.47-82.90 \%$ of isolates, C. glabrata in $5.6-30.09 \%$, C.famata in $0.08-2.20 \%$, C.krusei in $1.57-$ $11.17 \%$, C.parapsilosis in $0.49-11.30 \%$, C.tropicalis 2.43-7.8\%, unidentified Candida species in 0.90-7.54\%, Trichosporon species in $0.29-0.89 \%$ and S.cerevisiae in $0.15-0.70 \%$ of isolates. Most of the findings in this study were within these ranges except for C.famata, Trichosporon species and S. cerevisiae which were more frequent in this study. C.tropicalis was also isolated in these studies but was not found in the current study (7-14).

In the current study, flucytosine showed an overall susceptibility of $94.1 \% ; 5.0 \%$ of the isolates were resistant (Table 2). The tested strains demonstrated a $0-5.7 \%$ resistance except for C.krusei which had a resistance of $33.3 \%$. The high resistance rate in this species may be explained by the low rate of isolation of C. krusei (three isolates). Nawrot et al (13) found a low frequency $(0-3 \%)$ of resistance of tested strains while Richter et al (12) found an overall resistance of $3 \%$ with resistance of tested strains in the range $0-100 \%$. The $100 \%$ resistance was found in the strains isolated in small numbers in that study (12) C.lusitaniae and Trichosporon species which had one isolate each. A study on isolates of Cryptococcus neoformans from clinical sources from Nairobi found a $21.2 \%$ resistance to flucytosine (15). This is a drug for treatment of cryptococcosis and it has been recommended for use in combination with amphotericin B. It was not established whether that is actually being done in the current study. It was also not established whether the patients with flucytosine-resistant isolates had previously been on treatment with the drug.

Amphotericin B, which is the drug of choice for cryptococcal meningitis, demonstrated an overall susceptibility of $89.1 \%$ in this study, with $10.9 \%$ of isolates being resistant (Table 2). The susceptibility testing of this drug is still a controversial area; CLSI breakpoints have not yet been defined. Though some studies use a breakpoint of $1 \mu \mathrm{gml}^{-1}$, strains in this study with $\mathrm{MIC} \geq 2 \mu \mathrm{gml}^{-1}$ were considered resistant according to the manufacturer's instructions. There is limited information on amphotericin B susceptibility in this country except for cryptococcal infections. Biiet al (15) found a $0 \%$ resistance of the drug to C.neoformans with a low $\mathrm{MIC}_{90}$ of $0.5 \mu \mathrm{gml}^{-1}$. In the current study, though the resistance was much higher, a similar $\mathrm{MIC}_{90}$ was found for C.albicans with a resistance of $7.1 \%$; the other species had higher $\mathrm{MIC}_{90}$ values Table 2 of $1 \mathrm{\mu gml}^{-1}$ for non-albicans Candida (8\% resistant) and $16 \mathrm{gmml}^{-1}$ for non-candidal yeasts (66.7\% resistant). Other studies $(12,13,16)$ also demonstrated a low frequency of resistance to amphotericin $\mathrm{B}(0-1 \%)$. The use of amphotericin B for treatment of cryptococcal meningitis, which is an opportunistic infection that has increased especially in the wake of the HIV / AIDS pandemic, may result in development of resistancein the normal flora Candida species found in the vagina and in the mouth and if these then cause endogenous infection will demonstrate resistance to the drug. The resistant strains can also circulate in the community and may have far-reaching implications especially in the event of systemic candidiasis.

Fluconazole demonstrated an overall susceptibility of $93.1 \%$ and $3.0 \%$ resistance in the current study (Table 2). Among the isolates in this study, C. albicans had a $100 \%$ susceptibility which is in agreement with the results obtained in the study by Pfaller et al (8) that demonstrated C. albicans susceptibility of approximately $94-100 \%$ though with some geographical differences. In the same study, the C.albicans isolates from South Africa had a susceptibility of $99.4 \% \quad(0.3 \%$ resistant). In other studies, C.albicans demonstrated fluconazole susceptibility of $11(84.3 \%), 13(92 \%)$ and $14(92.5 \%)$. C.krusei has intrinsic resistance to fluconazole while C. glabrata shows reduced susceptibility. The C.glabrata isolates in the current study demonstrated a susceptibility of $84.6 \%$ (7.7\% S-DD, 7.7\% resistant). The ARTEMIS Global Antifungal Surveillance study demonstrated C.glabrata susceptibility in the range 29.2-100\%; the isolates from S. Africa had a susceptibility of $49.6 \%$ (21.1\% resistant). The $\mathrm{MIC}_{90}$ of fluconazole for C.albicans in the current study (Table 2) was $1 \mu \mathrm{gml}^{1}$ (range: 0.25-8) which was similar to that obtained by Comertet al (range: 0.125-8) (14). The $\mathrm{MIC}_{90}$ for C. glabrata in the current study was $32 \mu \mathrm{gml}^{-1}$ (range: 0.25-128) which was much higher than that for the same species in the study by Comert et al of $8 \mu \mathrm{gml}^{-1}$ (range: $0.125-16$ ). The elevated $\mathrm{MIC}_{90}$ for C.glabrata in this study may have been due to either the small number of C.glabrata isolates with a wide range of MIC distribution or to a rising resistance of the isolates in this study. Bii et al in the study on C.neoformans demonstrated a resistance to fluconazole of $11.2 \%$ with a $\mathrm{MIC}_{90}$ of $64 \mu \mathrm{gml}^{-1}(15)$. The $\mathrm{MIC}_{90}$ of C.neoformans in that study was higher than that of Candida in the current study hence C. neoformans demonstrated a higher resistance rate compared to Candida.

Itraconazolehad the lowest overall susceptibility (71.3\%) in this study with a resistance rate of $22.8 \%$ (Table 2). C.albicans was the species with the highest susceptibility $(90 \%)$ to this drug with that of the other isolates ranging between $0 \%$ (for C.parapsilosis which had only one isolate) and $100 \%$. The resistance rate for C. albicans was $10 \%$ which is in agreement with that found by Nawrot et al of 11\% (13). Non-albicans Candida had a resistance rate of $50 \%$ in the current study which was higher than that found by Galle et al of $23.5 \%$. The study by Bii showed itraconazole resistance rate of $6.3 \%\left(\mathrm{MIC}_{90} 0.5 \mu \mathrm{gml}^{-1}\right)$ which is much lower compared to that of Candidaisolatesin the current study. All the isolates resistant to fluconazole were 
also found to be resistant to itraconazole supporting the possibility of cross-resistance between the azoles. This cross-resistance may be due to a common mode of action of the azole derivatives.

Isolatesinhibited by fluconazole and itraconazole at concentrations of $16-32 \mu \mathrm{gml}^{-1}$ and 0.25 $0.5 \mu \mathrm{gml}^{-1}$ respectively are placed in a category known as susceptible-dose dependent (S-DD). This category identifies yeast isolates with intermediate susceptibilities, between fully susceptible and fully resistant. Isolates in this category will respond to fluconazole if higher doses are administered and to itraconazole if higher plasma levels are achieved (5). The definition of fluconazole susceptible and resistant does not apply for C. krusei sincethe organism is considered to have intrinsic resistance to the drug (6).

Identification of yeast isolates to species-level may aid in guiding the clinician on therapeutic options. VVC may be classified into complicated and uncomplicated forms. The uncomplicated form is caused by C.albicans, which is usually susceptible to all antifungal agents, and responds to short-course oral or topical treatment (17). The complicated form is recurrent, caused by non-albicans species of Candida and requires treatment for $\geq 7$ days. Patients with recurrent VVC are also more likely to acquire resistant Candida species due to frequent exposure to antifungal agents for the recurrent infections. Approximately $33 \%$ of RVVC is also known to be caused by nonalbicans Candida species some of which are inherently less susceptible to common antifungal agents making them less useful in their treatment (18). The optimal treatment of non-albicans Candida species remains unknown ${ }^{1}$ but they frequently respond to topical boric acid or topical flucytosine (17). Isolation of C.krusei in any specimen rules out the use of fluconazole for the treatment of the patient, even when the isolate tests susceptible in-vitro, since the species has intrinsic resistance to this antifungal agent while C.glabrata shows reduced susceptibility to the same agent, C.lusitaniae has been associated with amphotericin B resistance. Susceptibility testing is especially useful in infections due to non-albicans Candida species (17). In the setting of candidemia and deep infections susceptibility testing may be of benefit especially in cases where initial therapy has failed, the results may guide on suitable adjustment of therapy.

It is however important for the clinician to understand that in-vitro susceptibility results are supposed to be guidelines to the treatment of patients since they may not always predict the success of a particular treatment. However, when the infection is caused by a resistant isolate, the tests may predict the possibility of failure for a particular agent or the dosage. The clinician must also understand that in-vivo response to a drug is a combination of several factors: drug factors, host factors, site of the infection and fungal factors (5). Drug factors include the dosage (quantity, frequency and cumulative dose), pharmacokinetics (absorption, distribution, metabolism and excretion), pharmacodynamics and other concomitantly administered drugs (drug-drug interactions). Host factors include the immune system which works with the drug to clear the infection, the site and severity of the infection and patient compliance to the given treatment. Site of the infection factors include the source of the infection, drug penetration and the presence of a foreign body which acts as a foci for infection. Fungal factors include the species, cell type, the size of the micro-organism population, virulence factors, evasion of host inflammatory response and biofilm formation. All these factors have a role to play in the final resolution of the infection and should be taken into consideration when starting the patient on treatment.

The current study had limitations in time and cost. Due to these, a small sample size of 101 yeast isolates was analyzed. This resulted in some of the yeast species being isolated in very small numbers. The antifungal susceptibility testing was also to very few antifungal agents and none of the new agents in the market were tested for example voriconazole.

This study therefore paves the way for more studies with larger sample sizes, and in different parts of the country in order to understand the local epidemiology of yeast species and to study the local epidemiological susceptibility patterns. These would then be used to come up with local antibiograms that will aid clinicians in the selection of effective antifungal agents that can be used empirically. Larger studies to assess the clinical correlation of MIC and outcome are also required to enhance the understanding of the susceptibility testing in our set up. These studies would then form a baseline from which surveillance of antifungal drug resistance can be monitored both in hospitals and in the community.

In conclusion, this study observed that $C$. albicans was the prominent cause of vulvovaginal candidiasis in the study set up; non-albicans yeasts were a significant cause of vulvovaginal candidiasis. C.albicans still demonstrates susceptibility to fluconazole and itraconazole. Thereisahigherresistance demonstrated by non-albicans yeasts to fluconazole and itraconazole than by C.albicans.

Ethical issues: Ethical approval was obtained from the Ethical and Research Committee of the Aga Khan University Hospital, Nairobi.

\section{ACKNOWLEDGEMENTS}

To Prof. W. Jaoko, Dr. P. Odhiambo and Dr. C. Bii for taking time to read and for guidance on various aspects of the study; Management of Aga Khan 
University Hospital, Nairobi for allowing the use of their Microbiology laboratory;Aga KhanUniversity Hospital microbiology laboratory staff for theirimmensesupport and assistance, and Alice Lakati for data analysis.

\section{REFERENCES}

1. Centres for Disease Control and Prevention: Sexually Transmitted Diseases Treatment Guidelines 2002. Available on Internet from: http: / / www.cdc.gov/ STD/treatment/5-2002TG.htm (accessed 10February 2006).

2. Bauters, T.G., Dhont, M.A., Temmerman, M.I. and Nelis, H.J. Prevalence of vulvovaginal candidiasis and susceptibility to fluconazole in women. Amer. J. Obstet. Gyne. 2002; 187: 569-572.

3. Otero, L., Palacio, V., Carreno, F., et al. Vulvovaginal candidiasis in female sex workers. Intern J. STD/AIDS. 1998; 9: 526-530.

4. Sobel, J.D., Faro, S., Force, R.W., et al. Vulvovaginal candidiasis: Epidemiological, diagnostic and therapeutic considerations. Amer. J. Obstet. Gyne. 1998; 178: 203-211.

5. Hospenthal, D.R., Murray, C.K. and Rinaldi, M.G. The role of antifungal susceptibility testing in the therapy of candidiasis. Diag. Micro. Infect. Dis. 2004; 48: 153-160.

6. National Committee for Clinical Laboratory Standards. 2002. Reference method for broth dilution testing of yeasts. Approved standard - Second Edition. M27-A2. NCCLS, Wayne, Pa.

7. Spinillo, A., Capuzzo, E., Acciano, S., et al. Effect of antibiotic use on the prevalence of symptomatic vulvovaginal candidiasis. Amer. J. Obstet. Gyne. 1999; 180: 14-17.

8. Pfaller, M.A., Diekema, D.J., Rinaldi, M.G. et al and the Global Antifungal Surveillance Group. Results from the ARTEMIS DISK Global Antifungal Surveillance Study: a 6.5 year analysis of the susceptibilities of
Candida and other yeast species to fluconazole and voriconazole by standardized disk diffusion testing. J. Clin. Microb. 2005; 43: 5848-5859.

9. Namkinga, L.A., Matee, M.I.N, Kivaisi, K., et al. Identification of Candida strains isolated from Tanzanian pregnant women with vaginal candidiasis. East Afr. Med. J. 2005; 82: 226-234.

10. Verghese, S., Padmaya, P., Asha, M., et al. Prevalence, species distribution and antifungal sensitivity of vaginal yeast in infertile women. Indian J. Pathol. Microbiol. 2001; 44: 313 - 314.

11. Galle, L.C. and Gianinni, M.J.S.M. Prevalence and susceptibility of vaginal yeast. J. Brasileiro de Patologia e Medicina Laboratorial. 2004; 40: 229 - 236.

12. Richter, S.S., Galask, R.P., Messer, S.A., et al. Antifungal susceptibilities of Candida species causing vulvovaginitis and epidemiology of recurrent cases. J. Clin. Microb. 2005; 43: 2155-2162.

13. Nawrot, U., Nowicka, J., Juszczak, K., et al. Susceptibility to antifungal agents of Candida species isolated from paediatric and adult patients with haematological diseases. Mycoses. 2005; 48: 385-390.

14. Comert, F., Kulah, C., Aktas, E., et al. Identification of Candida species isolated from patients in intensive care unit and in vitro susceptibility to fluconazole for a 3 year period. Mycoses. 2006; 50: 52-57.

15. Bii, C.C., Makimura, K., Abe, S., et al. Antifungal drug susceptibility of Cryptococcus neoformons from clinical sources in Nairobi, Kenya. Mycoses. 2006; 50: 25-30.

16. Arikan, S. and Rex, J.H. Antifungal agents, in, Manual of Clinical Microbiology by Murray PR et al. ASM Press, Washington DC, USA. 2003; 2: 1859-1868.

17. Rex, J. H., Walsh, T. J., Sobel, J. D., et al. Practice guidelines for the treatment of Candidiasis. Clin. Infect. Dis. 2000; 30: 662-678.

18. Sobel, J.D. Pathogenesis and treatment of recurrent vulvovaginal candidiasis. Clin. Infect. Dis. 1992; 14: 148-153. 